\title{
Surgical approach to the thyroarytenoid branch of the inferior laryngeal nerve through the thyroid cartilage ${ }^{1}$
}

\author{
Fabiana Araújo SperandioI, Rui Imamura ${ }^{\text {II }}$, Domingos Hiroshi Tsuji ${ }^{\text {III }}$, Luiz Ubirajara Sennes ${ }^{\text {III }}$
}

DOI: http://dx.doi.org/10.1590/S0102-865020160070000003

${ }^{I} \mathrm{PhD}$, Supervisor, Residency Program in Otorhinolaryngology, Instituto de Medicina Integral Professor Fernando Figueira (IMIP), Recife-PE, Brazil. Scientific, intelectual, conception and design of the study; acquisition and interpretation of data; technical procedures; manuscript preparation and writing; final approval.

IPhD, Assistant Professor, Postgraduate Program in Otorhinolaryngology, Division of Otorhinolaryngology, School of Medicine, Universidade de São Paulo (USP), Brazil. Scientific and design of the study, statistical analysis, manuscript preparation and writing, critical revision, final approval.

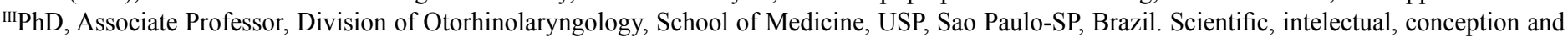
design of the study; manuscript preparation; critical revision; final approval.

\section{ABSTRACT}

PURPOSE: To describe the anatomical course of the intralaryngeal portion of the inferior laryngeal nerve (ILN) and to standardize the surgical access to its thyroarytenoid branch (TAb) through the thyroid cartilage.

METHODS: Under surgical microscopy, 33 adult human excised larynges were dissected, to expose the intralaryngeal portion of ILN. The point of entry of TAb, ILN's terminal branch, in the thyroarytenoid (TA) muscle was determined and correlated with thyroid cartilage dimensions.

RESULTS: After entering the larynx, the ILN consistently traveled between the thyroid cartilage and the lateral cricoarytenoid muscle in an anterior and slightly cranial course. The distance from the point of entry of the TAb in the TA muscle to the midline (TAb-H) and to the inferior border $(\mathrm{TAb}-\mathrm{V})$ of the thyroid cartilage differed according to gender. In females, mean distances of TAb-H and TAb-V were $20.5 \mathrm{~mm}$ and $5.2 \mathrm{~mm}$ and in males, $22.3 \mathrm{~mm}$ and $5.9 \mathrm{~mm}$, respectively.

CONCLUSION: The intralaryngeal course of the inferior laryngeal nerve presents low variability and measures from landmarks in the thyroid cartilage help to estimate the point of entry of thyroarytenoid branch in thyroarytenoid muscle.

Key words: Larynx. /anatomy \& histology. Laryngeal Mucosa. /innervation. 


\section{Introduction}

The thyroarytenoid (TA) muscle has a great impact in the biomechanics of the larynx and the physiology and pathophysiology of phonation. In adductor spasmodic dysphonia, dystonic contractions of the TA muscle during phonation leads to intermittent breaks in voicing, resulting in strained voice quality and dysfluent effortful speech ${ }^{1}$. Selective denervation of the TA muscle by sectioning the terminal thyroarynteoid branch (TAb) of the inferior laryngeal nerve (ILN) may improve dysphonia in these patients, while keeping other intrinsic laryngeal muscles intact ${ }^{2}$.

The TAb lies deep to the thyroid cartilage and the anatomy related to its surgical approach is not well known. Few studies describe the anatomy of the intralaryngeal portion of the inferior laryngeal nerve ${ }^{3-8}$, but the relation of TAb to anatomical landmarks of the thyroid cartilage is still poorly described.

We aimed to study the intralaryngeal portion of the inferior laryngeal nerve and standardize a surgical approach to the $\mathrm{TAb}$, through the thyroid cartilage.

\section{Methods}

The study was approved by the Ethics Committee of the Institution (nr. 266-98).

Thirty-three excised adult (18+yrs) human larynges (18 male, 15 female) from the Division of Postmortem Inspection of Universidade de São Paulo - School of Medicine were used in this study. Cases with any disease or malformation of the larynx or head and neck region were excluded. Larynges were fixed in $4 \%$ formalin for 7 days. The cricoarytenoid joint was preserved in all cases and the posterior aspect of the thyroid lamina, between the superior and inferior cornua, was ressected to create a semicircular window (around $1,5 \mathrm{~cm}$ radius) to expose the intralaryngeal branches of the anterior division of the ILN. The ILN was identified bilaterally in its hypopharyngeal portion and its anterior division was dissected upwards to its terminal branch, the TAb (Figure 1).

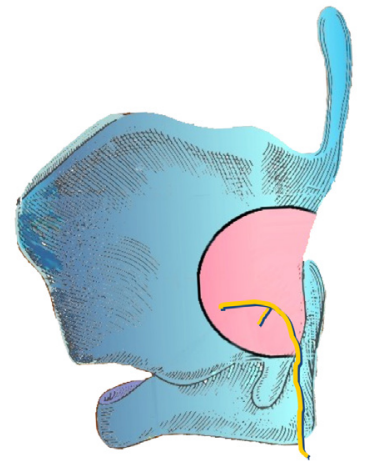

FIGURE 1 - Semicircular window resected at the posterior aspect of the thyroid lamina, between the superior and inferior cornua to expose the intralaryngeal portion of the inferior laryngeal nerve till its terminal thyroaryntenoid branch.
Nerve dissection was performed under an operating microscope at 6-x10 magnification. The projection of the anterior commissure of the glottis on the external midline of the thyroid cartilage was determined by a hypodermic $22 \mathrm{G}$ needle $(30 \mathrm{x}$ $0,7 \mathrm{~mm}$ ) placed horizontally through the anterior commissure. From this point, a line parallel to the plane of the inferior border of the thyroid cartilage was considered the level of the vocal fold (Figure 2), as defined by Isshiki ${ }^{9}$.

Larynges were dissected bilaterally. In each side, a surgical caliper (Factory, model F-199, Brazil) was used to obtain the following measures on the external surface of the thyroid cartilage (Figures 2 and 3):

Ht: Height of thyroid cartilage at midline, from the thyroid notch to the inferior border.

Ln: Length of the thyroid lamina at the vocal fold level.

Obl: Distance from midline to the oblique line of thyroid lamina at the vocal fold level.

TAb-H: Horizontal distance from midline of thyroid cartilage to the point of entry of TAb in TA muscle.

$\mathrm{TAb}-\mathrm{V}$ : Vertical distance from inferior border of thyroid cartilage to the point of entry of TAb in TA muscle.

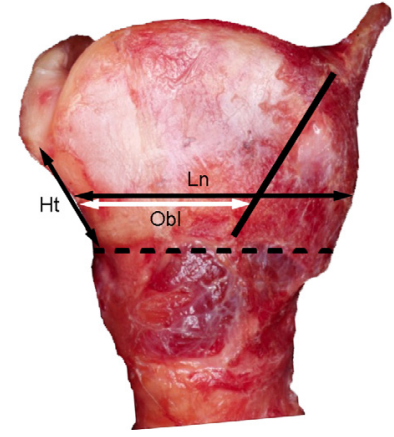

FIGURE 2 - Measurements made on thyroid cartilage. Ht: height of thyroid cartilage at midline, from the thyroid notch to the inferior border; Ln: Length of the thyroid lamina at the vocal fold level; Obl: Distance from midline to the oblique line (black thick line) of thyroid lamina at the vocal fold level. Dashed line: plane that determines the inferior border of thyroid cartilage.

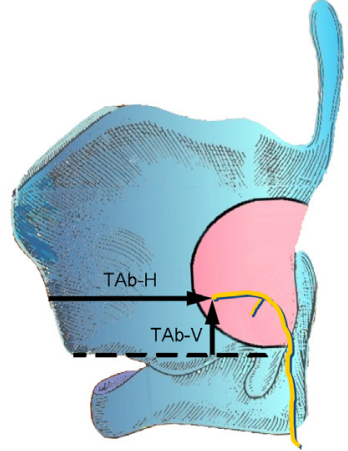

FIGURE 3 - Measurements made on the thyroid cartilage to determine the point of entry of the thyroarytenoid branch (TAb) of the inferior laryngeal nerve in the thyroarytenoid (TA) muscle. TAb-H: Horizontal distance from midline of thyroid cartilage to the point of entry of TAb in TA muscle; TAb-V: Vertical distance from the plane that determines the inferior border of thyroid cartilage (dashed line) to the point of entry of $\mathrm{TAb}$ in TA muscle. 
Subjective analysis of histogram and Shapiro-wilk normality test suggested normal distribution of the data and parametric tests were used. Differences according to gender and side of the larynx were compared with unpaired and paired Student $t$ tests, respectively. Pearson correlation coefficient was used in univariate analyses to seek for associations between the variables TAb-H and TAb-V and laryngeal dimensions (Ht, Ln and $\mathrm{Obl}$ ). Multivariate analyses were performed to estimate the variables $\mathrm{TAb}-\mathrm{H}$ and $\mathrm{TAb}-\mathrm{V}$ in each side based on gender and other laryngeal dimensions. The study was considered exploratory, with no primary question and no sample size calculation. Level of significance (alpha) of 5\% was adopted.

\section{Results}

In all 66 hemi larynges, the intralaryngeal course of the anterior division of the ILN was consistent, with no significant variance. Ascending lateral to the posterior cricoarytenoid (PCA), it gave branches to this muscle and to the interarytenoid (IA) muscle and curved anteriorly, over the cricothyroid articulation, passing $1-2 \mathrm{~mm}$ of the joint. It then moved in an anterior and slightly cranial direction, in the paraglottic space. In 60 cases, the nerve travelled between the lateral cricoarytenoid (LCA) muscle and the inner pericondrium of the thryoid lamina and in 6 cases, it passed through the LCA muscle before the TAb entered the TA muscle. A unique branch to LCA was found in 37 dissections (56\%), bipedicular innervation in 23 cases $(35 \%)$ and three branches in six cases $(9 \%)$. In all cases, TAb entered the posterior third of TA muscle, anterior to the oblique line of the thyroid lamina (Figure 4). The nerve ending of TAb in TA muscle was like a brush in 58 cases $(88 \%)$ and as a unique branch in 8 cases $(12 \%)$.

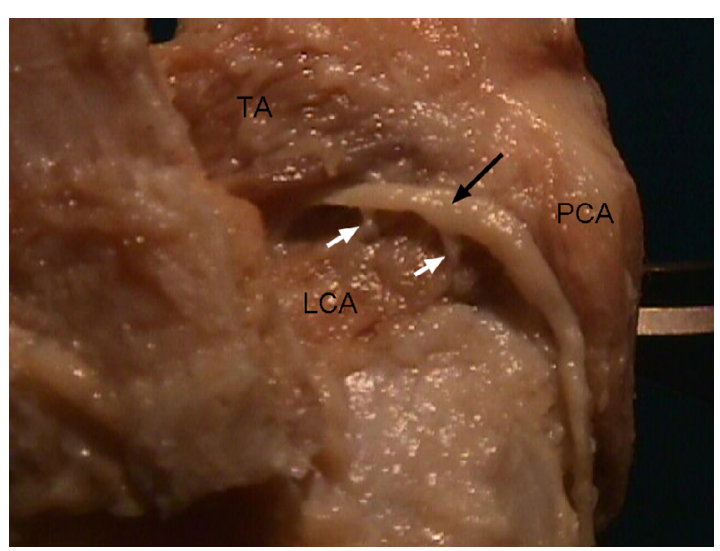

FIGURE 4 - Left hemi larynx showing intralaryngeal course of ILN (black arrow). Point of entry of the thyroarytenoid branch (TAb) in the thyroarytenoid (TA) muscle as a unique branch, with two branches (white arrows) to the lateral cricoarytenoid (LCA) muscle. PCA: posterior cricoarytenoid muscle.

The mean and 99\% confidence interval (CI) of each of the laryngeal dimensions and measures according to gender are depicted in Table 1. Mean values were always statistically larger in male larynges. Table 2 shows values related to $\mathrm{Ln}, \mathrm{Obl}$, TAb-H and $\mathrm{TAb}-\mathrm{V}$ in each side of the larynx.

TABLE 1 - Laryngeal dimensions and coordinates to identify the point of entry of TAb in TA muscle, according to gender.

\begin{tabular}{|c|c|c|c|c|c|c|c|}
\hline \multirow[b]{2}{*}{ Variable } & \multicolumn{3}{|c|}{ Female $(n=15)$} & \multicolumn{3}{|c|}{ Male $(n=18)$} & \multirow[b]{2}{*}{$\mathrm{p}(1)$} \\
\hline & Mean & $\begin{array}{l}99 \% \mathrm{CI} \text { inferior } \\
\text { limit }\end{array}$ & $\begin{array}{c}99 \% \text { CI superior } \\
\text { limit }\end{array}$ & Mean & $\begin{array}{l}99 \% \mathrm{CI} \text { inferior } \\
\text { limit }\end{array}$ & $\begin{array}{l}99 \% \text { CI superior } \\
\text { limit }\end{array}$ & \\
\hline $\mathrm{Ht}$ & 17.733 & 15.69 & 19.777 & 20.056 & 18.92 & 21.191 & 0.0045 \\
\hline $\operatorname{Ln}(\mathrm{R})$ & 35.733 & 33.732 & 37.735 & 37.722 & 36.532 & 38.913 & 0.0136 \\
\hline $\operatorname{Ln}(\mathrm{L})$ & 35.866 & 33.563 & 38.17 & 37.944 & 36.63 & 39.259 & 0.0221 \\
\hline Obl (R) & 31.533 & 29.893 & 33.173 & 33.389 & 32.081 & 34.696 & 0.0131 \\
\hline Obl (L) & 32.0666 & 30.196 & 33.937 & 33.778 & 32.593 & 34.962 & 0.0251 \\
\hline TAb-H (Rt) & 20.533 & 19.668 & 21.398 & 22.278 & 21.439 & 23.116 & 0.0002 \\
\hline TAb-H (Lt) & 20 & 18.734 & 21.266 & 22.333 & 21.522 & 23.145 & $<0.0001$ \\
\hline TAb-V (Rt) & 5.2 & 4.68 & 5.72 & 5.889 & 5.427 & 6.351 & 0.0066 \\
\hline $\mathrm{TAb}-\mathrm{V}(\mathrm{Lt})$ & 5.133 & 4.642 & 5.625 & 5.944 & 5.576 & 6.313 & 0.0004 \\
\hline
\end{tabular}

TAb: thyroarytenoid branch of inferior laryngeal nerve; TA: thyroarytenoid; CI: confidence interval; Ht: height of the thyroid cartilage at midline; Ln and Obl: length of the thyroid lamina and distance from midline to oblique line at vocal fold level;

TAb-H: horizontal distance from midline of thyroid cartilage to the point of entry of TAb in TA muscle; TAb-V: vertical distance from inferior border of thyroid cartilage to the point of entry of TAb in TA muscle; Rt: right; Lt: left; (1) Student t test, unpaired 
TABLE 2 - Laryngeal dimensions according to hemi larynx side.

\begin{tabular}{|c|c|c|c|c|c|c|c|}
\hline \multirow[b]{2}{*}{ Variable } & \multirow[b]{2}{*}{ Mean } & \multicolumn{2}{|c|}{ Right (n=33) } & \multicolumn{3}{|c|}{ Left $(n=33)$} & \multirow[b]{2}{*}{$\mathrm{p}(1)$} \\
\hline & & $95 \% \mathrm{CI}$ inferior limit & $95 \% \mathrm{CI}$ superior limit & Mean & $95 \% \mathrm{CI}$ inferior limit & $95 \% \mathrm{CI}$ superior limit & \\
\hline Ln & 36.818 & 35.979 & 37.656 & 37 & 36.061 & 37.938 & 0.4393 \\
\hline Obl & 32.545 & 31.767 & 33.323 & 33 & 32.212 & 33.787 & 0.0113 \\
\hline TAb-H & 21.484 & 20.967 & 22.002 & 21.272 & 20.625 & 21.919 & 0.2137 \\
\hline TAb-V & 5.575 & 5.309 & 5.842 & 5.575 & 5.324 & 5.826 & 1 \\
\hline
\end{tabular}

CI: confidence interval; Ln and Obl: length of the thyroid lamina and distance from midline to oblique line at vocal fold level; TAb-H: horizontal distance from midline of thyroid cartilage to the point of entry of TAb in TA muscle; TAb-V: vertical distance from inferior border of thyroid cartilage to the point of entry of TAb in TA muscle; (1) Student $t$ test, paired

Table 3 shows the correlation matrix between the measures TAb- $\mathrm{H}$ and $\mathrm{TAb}-\mathrm{V}$ in each side and laryngeal dimensions (Ht, Obl, Ln).

TABLE 3 - Pearson's correlation coefficient (r) between laryngeal measures (TAb-H and TAb-V) and dimensions (Ht, Obl, $\mathrm{Ln})$.

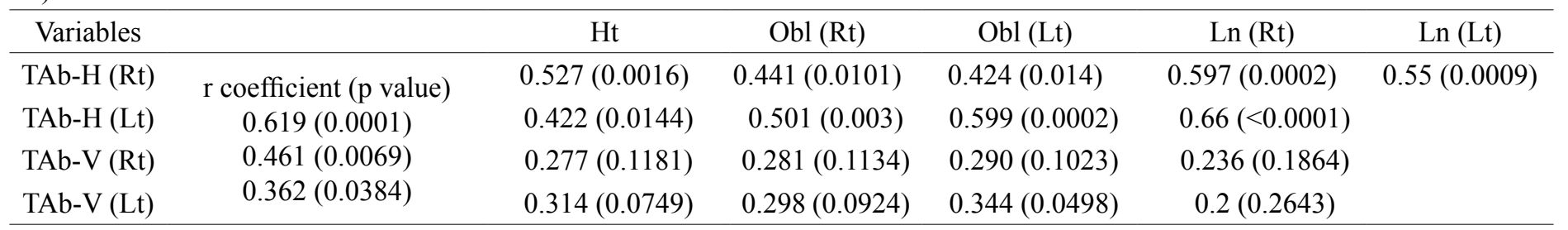

$\mathrm{Ht}$ : height of the thyroid cartilage at midline; Ln and Obl: length of the thyroid lamina and distance from midline to oblique line at vocal fold level; Rt: right; Lt: left; $\mathrm{TAb}-\mathrm{H}$ : horizontal distance from midline of thyroid cartilage to the point of entry of TAb in TA muscle; TAb-V: vertical distance from inferior border of thyroid cartilage to the point of entry of TAb in TA muscle.

Data on multiple linear regression models to estimate TAb-H e TAb-V in each side are shown in Table 4. For TAb-H, relevant predictors were gender and Ln. In the right side, the formula: $11.384+1.235^{*}$ Gender $+0.256 *$ Ln could be used to estimate TAb-H with a model fit around $50 \%$. In the left side, the formula: $8.151+1.645 *$ Gender $+0.33 * \mathrm{Ln}$ could be used to estimate TAb-H with a model fit around $60 \%$.

TABLE 4 - Multiple linear regression models to estimate point of entry of TAb in TA muscle.

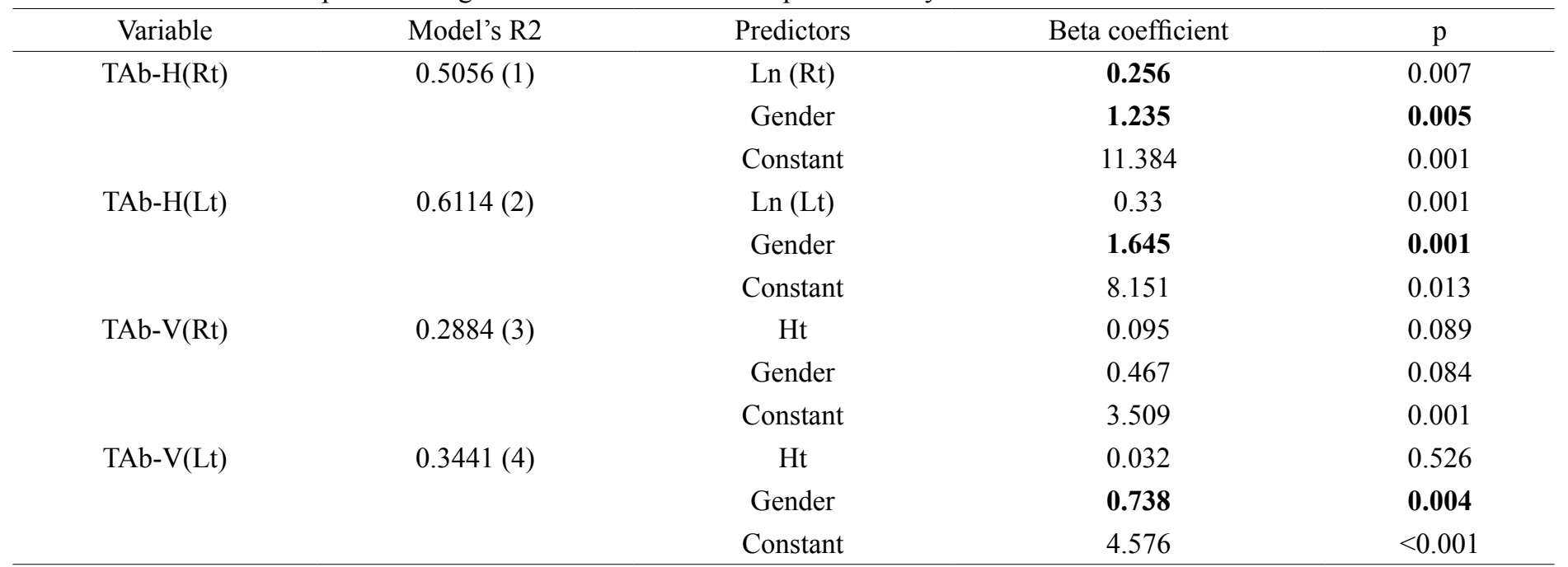

TAb: thyroarytenoid branch of inferior laryngeal nerve; TA: thyroarytenoid; R2: coefficient of determination; TAb-H: horizontal distance from midline of thyroid cartilage to the point of entry of TAb in TA muscle; TAb-V: vertical distance from inferior border of thyroid cartilage to the point of entry of TAb in TA muscle; Ht: height of the thyroid cartilage at midline; Ln and Obl: length of the thyroid lamina and distance from midline to oblique line at vocal fold level; Rt: right; Lt: left;

(1) $\mathrm{Ht}, \mathrm{Obl}(\mathrm{Rt})$ : n.s. when added to the model and did not contribute to significant increase in R2

(2) $\mathrm{Ht}, \mathrm{Obl}(\mathrm{Lt})$ : n.s. when added to the model and did not contribute to significant increase in R2

(3) Obl (Rt), Ln (Rt): n.s. when added to the model and did not contribute to significant increase in R2

(4) $\mathrm{Obl}(\mathrm{Lt}), \mathrm{Ln}(\mathrm{Lt})$ : n.s. when added to the model and did not contribute to significant increase in R2 


\section{Discussion}

Precise anatomical knowledge of intralaryngeal structures in relation to the laryngeal framework allows the surgeon to access neuromuscular elements and interfere in pathophysiological conditions, without directly disturbing in the delicate mucosa of the vocal tract ${ }^{9}$.

In this study, we propose a surgical access to the TAb of ILN through the thyroid cartilage, that may be used in patients with adductor spasmodic dysphonia ${ }^{10-12}$ or other pathophysiological conditions that involve the TA muscle. Chemical denervation of the TA muscle with botulinum toxin injection is currently the treatment of choice of adductor spasmodic dysphonia in most centers, but as the effect is time-limited, it requires periodic injections ${ }^{13}$. Furthermore, patients usually refer vocal instability at the beginning and at the end of drug action. Therefore, surgical denervation may be advantageous as it may offer a more definitive result $^{10,12}$. Severing of extra-laryngeal ILN has been proposed for treating this condition, but although it is easier to perform, it results in more morbidity due to the denervation of other intrinsic laryngeal muscles ${ }^{14}$.

No anatomical variations or anomalies in the intralaryngeal course of the ILN were detected in this study. The nerve path was consistent in all specimens and agreed with former descriptions $^{3,4,7}$.

Morphometric studies of adult human larynges emphasize differences according to gender, but not in regard to somatotype or age $^{9,15-17}$. Therefore, only gender was considered in the analysis of our results. In a given gender the point of entry of TAb in the TA muscle, defined by the measures TAb-H and TAb-V, showed little variance in our study, even adopting strict $99 \%$ CIs. That means the point of entry of TAb in the TA muscle can be predicted by laryngeal landmarks and its surgical trans-thyroid access does not require extensive dissection from the extra-laryngeal ILN or large resections of the thyroid cartilage as performed in this anatomical study. We believe that a distal access to the specific branch of interest through a small window opened in the thyroid lamina is a better approach as the lesser the nerve is manipulated the lower the risk of unnecessary nerve damage.

Univariate analyses found positive significant correlations between $\mathrm{TAb}-\mathrm{H}$ and $\mathrm{TAb}-\mathrm{V}$ and the laryngeal dimensions $\mathrm{Ht}$, Obl and Ln (Table 3). Although we recognize these analyses as exploratory, the highest correlation for $\mathrm{TAb}-\mathrm{H}$ was with measure $\mathrm{Ln}$. For TAb-V in both sides, the highest correlation was with measure Ht. These findings suggest that the point of entry of TAb in TA muscle follows a given proportion with both vertical and horizontal dimensions of the thyroid cartilage.
In our study, we noticed that TAb always entered the TA muscle anteriorly to the oblique line of the thyroid lamina. That is in accordance to previous anatomical studies ${ }^{8,15}$. However, the oblique line did not seem to be a good anatomical reference to identify TAb, as suggested by other authors ${ }^{8}$. As demonstrated in Table 2, although most laryngeal measures (Ln, TAb-H and $\mathrm{TAb}-\mathrm{V})$ presented with fairly good symmetry between sides, the same did not occur with Obl, suggesting that the oblique line does not hold a constant relation with other laryngeal dimensions. Furthermore, TAb-H in each side of the larynx showed a stronger correlation with the measure $\mathrm{Ln}$, than with $\mathrm{Obl}$ in both univariate and multivariate analyses (Tables 3 and 4).

In a surgical setting for selective denervation of the TAb, the nerve branch should be identified prior to the point of entry in TA muscle, to allow proper space for sectioning. As the course of the TAb is anterior and slightly cranial towards the TA muscle, we propose centering the trans-thyroid access window in the upper limit of the $99 \%$ CI for TAb-H and the lower limit of $99 \% \mathrm{CI}$ for TAb-V. According to our results (Table 1), distances from the center of the window to the midline and the plane that determines the inferior border of the thyroid cartilage should be, respectively, $21.4 \mathrm{~mm}$ and $4.6 \mathrm{~mm}$ in females and $23.1 \mathrm{~mm}$ and $5.4 \mathrm{~mm}$, in males. The size of the window should be tailored according to the surgeon's needs for proper handling of the nerve branch, but we believe a window as small as $5 \times 4 \mathrm{~mm}$ should suffice to locate and section the TAb of ILN (Figure 5).

Concerning external validity of our data, we have limited our sample to adults (18 years old or older), which usually constitutes the target population in surgical candidates with adductor spasmodic dysphonia. In a large series of such patients, the age of onset was $39 \pm 16$ years $^{13}$. We know that anthropometric measurements may vary according to the study population, which may limit generalizability of results in any anatomical study. We did no restrict our sample according to race and despite the large race heterogeneity of Brazilian population, we did not find large variances in TAb-H and TAb-V, suggesting small ethnic influence in these measurements. However, our results should be confirmed in other populations to allow generalization of findings.

As described by Isshiki ${ }^{9}$ and according to our results, we believe that the laryngeal framework should not be considered a physical barrier to phonosurgical procedures and that neuromuscular elements can be surgically manipulated in order to correct laryngeal pathophysiological conditions. Besides the selective surgical denervation of TA muscle, we believe the described access to the TAb may be used in the future for specific laryngeal reinnervation procedures. 


\section{Conclusions}

The course of the intralaryngeal portion of the laryngeal nerve (ILN) was consistent: the anterior division of ILN enters the endolarynx 1-2mm over the cricothyroid joint and runs anterior and slightly cranially in the paraglottic space, giving branches to the lateral cricoarytenoid (LCA) muscle before penetrating the posterior third of the thyroarytenoid (TA) muscle, anterior to the oblique line. There were significant differences in laryngeal dimensions according to gender, but not in relation to laryngeal side, except for Obl.

In a given gender, the point that the thyroarytenoid branch (Tab) enters the TA muscle, defined by coordinates TAb-H and $\mathrm{TAb}-\mathrm{V}$, showed little variance, suggesting that they can be used for the trans-thyroid identification of TAb.

\section{References}

1. Nash EA, Ludlow CL. Laryngeal muscle activity during speech breaks in adductor spasmodic dysphonia. Laryngoscope. 1996 Apr;106(4):484-9. PMID: 8614226.

2. Berke GS, Blackwell KE, Gerratt BR, Verneil A, Jackson KS, Sercarz JA. Selective laryngeal adductor denervation-reinnervation: a new surgical treatment for adductor spasmodic dysphonia. Ann Otol Rhinol Laryngol. 1999 Mar;108(3):227-31. PMID: 10086613.

3. Nguyen M, Junien-Lavillauroy C, Faure C. Anatomical intralaryngeal anterior branch study of the recurrent (inferior) laryngeal nerve. Surg Radiol Anat. 1989;11(2):123-7. PMID: 2763003.

4. Schweizer V, Dörfl J. The anatomy of the inferior laryngeal nerve. Clin Otolaryngol Allied Sci. 1997 Aug;22(4):362-9. PMID: 9298614.

5. Sanders I, Wu BL, Mu L, Li Y, Biller HF. The innervation of the human larynx. Arch Otolaryngol Head Neck Surg. 1993 Sep;119(9):934-9. PMID: 7689327.

6. Sanudo JR, Maranillo E, Leon X, Mirapeix RM, Orus C, Quer M. An anatomical study of anastomoses between the laryngeal nerves. Laryngoscope. 1999 Jun;109(6):983-7. PMID: 10369294.

7. Prades JM, Faye MB, Timoshenko AP, Dubois MD, Dupuis-Cuny A, Martin C. Microsurgical anatomy of intralaryngeal distribution of the inferior laryngeal nerve. Surg Radiol Anat. 2006 Jun;28(3):2716. PMID: 16612555.

8. Scheid SC, Nadeau DP, Friedman O, Sataloff RT. Anatomy of the thyroarytenoid branch of the recurrent laryngeal nerve. J Voice. 2004 Sep;18(3):279-84. PMID: 15331099.
9. Isshiki N. Phonosurgery: theory and practice. New York: SpringerVerlag; 1989.

10. Tsuji DH, Takahashi MT, Imamura R, Hachiya A, Sennes LU. Endoscopic laser thyroarytenoid myoneurectomy in patients with adductor spasmodic dysphonia: a pilot study on long-term outcome on voice quality. J Voice. 2012 Sep;26(5):666 e7-12. PMID: 21940146

11. Ludlow CL. Treatment for spasmodic dysphonia: limitations of current approaches. Curr Opin Otolaryngol Head Neck Surg. 2009 Jun;17(3):160-5. PMID: 19337127.

12. Su CY, Lai CC, Wu PY, Huang HH. Transoral laser ventricular fold resection and thyroarytenoid myoneurectomy for adductor spasmodic dysphonia: long-term outcome. Laryngoscope. 2010 Feb;120(2):313-8. PMID: 20013850.

13. Blitzer A, Brin MF, Stewart CF. Botulinum toxin management of spasmodic dysphonia (laryngeal dystonia): a 12-year experience in more than 900 patients. Laryngoscope. 2015 Aug;125(8):1751-7. PMID: 26200329.

14. Dedo HH. Recurrent laryngeal nerve section for spastic dysphonia. Ann Otol Rhinol Laryngol. 1976 Jul-Aug;85(4 Pt 1):451-9. PMID: 949152.

15. Meiteles LZ, Lin PT, Wenk EJ. An anatomic study of the external laryngeal framework with surgical implications. Otolaryngol Head Neck Surg. 1992 Mar;106(3):235-40. PMID: 1589212.

16. Friedrich G, Lichtenegger R. Surgical anatomy of the larynx. J Voice. 1997 Sep;11(3):345-55. PMID: 9297680.

17. Jotz GP, Stefani MA, Pereira da Costa Filho O, Malysz T, Soster PR, Leao HZ. A morphometric study of the larynx. J Voice. 2014 Nov;28(6):668-72. PMID: 24814367.

\section{Correspondence:}

Rui Imamura

Avenida Enéas de Carvalho Aguiar, 255

05403-000 São Paulo - SP Brasil

Tel.: (55 11)3088-0299

imamurar@terra.com.br

Received: Mar 10, 2016

Review: May 12, 2016

Accepted: Jun 13, 2016

Conflict of interest: none

Financial source: FAPESP

${ }^{1}$ Research performed at Medical Investigation Laboratory (LIM-32), Division of Otorhinolaryngology, School of Medicine, Universidade de São Paulo, Brazil. Part of $\mathrm{PhD}$ degree thesis, Postgraduate Program in Otorhinolaryngology. Tutor: Luiz Ubirajara Sennes. 